\title{
Chaos Control and Synchronization via Switched Output Control Strategy
}

\author{
Runzi Luo, Haipeng Su, and Yanhui Zeng \\ Department of Mathematics, Nanchang University, Nanchang 330031, China \\ Correspondence should be addressed to Runzi Luo; luo_rz@163.com
}

Received 24 October 2016; Accepted 22 December 2016; Published 29 January 2017

Academic Editor: Francisco Gordillo

Copyright (C) 2017 Runzi Luo et al. This is an open access article distributed under the Creative Commons Attribution License, which permits unrestricted use, distribution, and reproduction in any medium, provided the original work is properly cited.

\begin{abstract}
This paper investigates the control and synchronization of a class of chaotic systems with switched output which is assumed to be switched between the first and the second state variables of chaotic system. Some novel and yet simple criteria for the control and synchronization of a class of chaotic systems are proposed via the switched output. The generalized Lorenz chaotic system is taken as an example to show the feasibility and efficiency of theoretical results.
\end{abstract}

\section{Introduction}

Chaotic phenomenon occurs naturally in many engineering, physical, biological, and social systems [1]. Although chaotic phenomenon could be beneficial in some applications, it is undesirable in many physical applications and should therefore be controlled in order to improve the system performance. For a quite long period of time, due to the high sensitivity of a chaotic system to its initial condition, people thought that chaos was not controllable, and two chaotic systems could not be synchronized. However, the OGY method [2] developed by Ott et al. and in particular the concept of the synchronization proposed by Pecora and Carroll [3] in 1990 have completely changed the situation. Control and synchronization of chaotic systems have many potential applications in physical systems, lasers, circuits, chemical reactor, ecological systems, and secure communication $[1,4]$. For example, in the field of secure communication many chaotic systems, such as the logistic map, the Hénon map, and the piecewise linear chaotic map [5], have been used to develop chaotic ciphers due to the sensitivity to initial conditions, ergodicity, and pseudorandom behavior of chaotic systems satisfies the analogous requirements for a good cryptosystem [6]. Recently, spatiotemporal chaos has been employed for hash functions [7] and Chebyshev maps have been used for key agreement protocols [8].

Owing to the potential applications of chaos control and synchronization, many efforts have been devoted by researchers to achieve the goals of chaos control and synchronization in the last two decades and, as a result, a wide variety of approaches have been proposed for the control and synchronization of chaotic systems which include adaptive control [9], active control [10], integral control [11], impulsive control [12], backstepping control [13], sampled-data control [14], and so forth.

In the literature there are many results concerning the control and synchronization of chaotic system [3, 9-21]. However, most of the existing works dealing with controlling chaos and chaos synchronization are based on the same assumption that the state variables of chaotic systems are all available for designing the controller. As it is well known that for most nonlinear systems the state variables are often unavailable in practice. For example, in the input-output system only the output is available which means that the above requirement is not very reasonable. Thus, the investigation of chaos control and synchronization with only output states available becomes an important topic. On the other hand, the transmitted signal may be switched between different signals and even interrupted for a variety of reasons. Therefore, it is necessary and important to investigate the control and synchronization of chaotic system with switched output. So far as we know, less attention has been paid to this issue.

With the above motivations, our main aim in this paper is to investigate the control and synchronization of a class of chaotic systems with switched output. The chaotic systems 
are assumed that only the output variable is available and the output may be switched between the first and the second state variables. Some novel criteria for the control and synchronization of a class of chaotic systems are proposed via the switched output. The generalized Lorenz chaotic system is taken as an example to show the feasibility and efficiency of theoretical results.

The paper is organized as follows. First, a brief description of a class of chaotic systems is introduced in Section 2. The control and the synchronization schemes are discussed in Sections 3 and 4, respectively. Section 5 includes several numerical examples to demonstrate the effectiveness of the proposed approach. Finally, conclusion remarks are presented in Section 6.

\section{System Description}

Consider the following chaotic system:

$$
\begin{aligned}
& \dot{x}_{1}=a\left(x_{2}-x_{1}\right), \\
& \dot{x}_{2}=b x_{1}+c x_{2}-x_{1} x_{3}, \\
& \dot{x}_{3}=x_{1} x_{2}-d x_{3}, \\
& x_{\text {out }}=k_{1}(t) x_{1}+k_{2}(t) x_{2}, \\
& k_{1}(t) \\
& \quad= \begin{cases}1, & t \in\left[t_{2 m}, t_{2 m+1}\right), t_{0}=0, m=0,1,2, \ldots, \\
0, & t \in\left[t_{2 m+1}, t_{2 m+2}\right), m=0,1,2, \ldots,\end{cases} \\
& k_{2}(t) \quad \begin{array}{ll}
0, & t \in\left[t_{2 m}, t_{2 m+1}\right), t_{0}=0, m=0,1,2, \ldots, \\
1, & t \in\left[t_{2 m+1}, t_{2 m+2}\right), m=0,1,2, \ldots,
\end{array}
\end{aligned}
$$

where $x=\left(x_{1}, x_{2}, x_{3}\right)^{T} \in R^{3 \times 1}$ is the state vector of system (1) and $a(\neq 0), b, c(<0)$, and $d(>0)$ are the system's parameters, which are known in advance. $x_{\text {out }}$ denotes the output variable of system (1).

Remark 1. It is well known that some chaotic systems such as the Lorenz system [22], the generalized Lorenz system [23], and the unified chaotic system [24] can be written in the form of system (1).

Let $\left|t_{i} t_{i+1}\right|$ denote the length of interval $\left[t_{i}, t_{i+1}\right)$ or $\left[t_{i}, t_{i+1}\right)$. Furthermore, we suppose that $\alpha_{m}=\left|t_{2 m} t_{2 m+1}\right|$, $\beta_{m}=\left|t_{2 m+1} t_{2 m+2}\right|, \alpha_{\min }=\min \left\{\alpha_{m}\right\}, \alpha_{\max }=\max \left\{\alpha_{m}\right\}, \beta_{\min }=$ $\min \left\{\beta_{m}\right\}$, and $\beta_{\max }=\max \left\{\beta_{m}\right\}$, where $m=0,1,2, \ldots, 0 \leq$ $\alpha_{\max }<\infty, 0 \leq \beta_{\max }<\infty$, and $\alpha_{\max }, \beta_{\max }$ are not both zero.

Remark 2. It is easy to see that if $x_{\text {out }}=x_{1}$ or $x_{\text {out }}=x_{2}$, then the output $x_{\text {out }}$ of system (1) is continuous variable. Otherwise, the output $x_{\text {out }}$ is switched variable which is discontinuous.

\section{The Control Scheme of a Class of Chaotic Systems}

In this section, we investigate the stabilization of system (1) at origin. For the purpose of forcing the states to converge to origin, we add two controllers to system (1). The controlled system (1) with a specified output is given as

$$
\begin{aligned}
& \dot{x}_{1}=a\left(x_{2}-x_{1}\right)+u_{1}, \\
& \dot{x}_{2}=b x_{1}+c x_{2}-x_{1} x_{3}+u_{2}, \\
& \dot{x}_{3}=x_{1} x_{2}-d x_{3}, \\
& x_{\text {out }}=k_{1}(t) x_{1}+k_{2}(t) x_{2}, \\
& k_{1}(t) \\
& \quad= \begin{cases}1, & t \in\left[t_{2 m}, t_{2 m+1}\right), t_{0}=0, m=0,1,2, \ldots, \\
0, & t \in\left[t_{2 m+1}, t_{2 m+2}\right), m=0,1,2, \ldots,\end{cases} \\
& k_{2}(t) \quad \begin{array}{ll}
0, & t \in\left[t_{2 m}, t_{2 m+1}\right), t_{0}=0, m=0,1,2, \ldots, \\
1, & t \in\left[t_{2 m+1}, t_{2 m+2}\right), m=0,1,2, \ldots,
\end{array}
\end{aligned}
$$

where $u_{1}$ and $u_{2}$ are the controllers to be designed later.

Our work in this section is to design controller $u(t)=$ $u\left(x_{\text {out }}(t)\right)$ to make system (2) be stabilized at origin; that is, $\lim _{t \rightarrow+\infty} x_{1}=\lim _{t \rightarrow+\infty} x_{2}=\lim _{t \rightarrow+\infty} x_{3}=0$.

Assumption 3. Suppose the state variables of system (2) are bounded, which means that there exists nonnegative constant $M$ such that $\left|a\left(b x_{1}+c x_{2}-x_{1} x_{3}\right)+a(1-a)\left(x_{2}-x_{1}\right)-\ddot{x}_{1}+x_{1}\right| \leq$ $M$.

Theorem 4. For system (2), suppose

$$
\begin{aligned}
& u_{1}=-a k_{2}(t) x_{\text {out }}, \\
& u_{2}=-k_{1}(t) \operatorname{sgn}\left(x_{\text {out }}\right) \frac{M}{a} ;
\end{aligned}
$$

then we have the following:

(1) If $\alpha_{\max }=\infty$, then $\lim _{t \rightarrow+\infty} x_{1}=\lim _{t \rightarrow+\infty} x_{2}=$ $\lim _{t \rightarrow+\infty} x_{3}=0$.

(2) If $\beta_{\max }=\infty$ and $a>0$, then $\lim _{t \rightarrow+\infty} x_{1}=$ $\lim _{t \rightarrow+\infty} x_{2}=\lim _{t \rightarrow+\infty} x_{3}=0$.

(3) If $\alpha_{\max } \beta_{\max } \neq \infty, a<0$, and there exists constant $\varepsilon>0$ such that $\alpha_{\min }+a \beta_{\max } \geq \varepsilon$, then $\lim _{t \rightarrow+\infty} x_{1}=$ $\lim _{t \rightarrow+\infty} x_{2}=\lim _{t \rightarrow+\infty} x_{3}=0$.

(4) If $\alpha_{\max } \beta_{\max } \neq \infty, a>0$, then $\lim _{t \rightarrow+\infty} x_{1}=$ $\lim _{t \rightarrow+\infty} x_{2}=\lim _{t \rightarrow+\infty} x_{3}=0$.

Proof. Note that $c<0$; from the second equation of system (2) and the expression of $u_{1}$ and $u_{2}$ it is easy to see that if $\lim _{t \rightarrow+\infty} x_{1}=0$ then $\lim _{t \rightarrow+\infty} x_{2}=0$. Keep in mind that $d>$ 0 ; from the third equation of system (2) it is obvious that if $\lim _{t \rightarrow+\infty} x_{1}=\lim _{t \rightarrow+\infty} x_{2}=0$ then $\lim _{t \rightarrow+\infty} x_{3}=0$. Thus, in 
order to prove $\lim _{t \rightarrow+\infty} x_{1}=\lim _{t \rightarrow+\infty} x_{2}=\lim _{t \rightarrow+\infty} x_{3}=0$ we only need to show that $\lim _{t \rightarrow+\infty} x_{1}=0$. Two cases are discussed in the following according to $t$.

Case 1. If $t \in\left[t_{2 m}, t_{2 m+1}\right), m=0,1,2, \ldots$, then $k_{1}(t)=1$ and $k_{2}(t)=0$. From the first equation of system (2), we have

$$
\begin{aligned}
\dot{x}_{1} & =a\left(x_{2}-x_{1}\right) \\
\ddot{x}_{1} & =a\left(\dot{x}_{2}-\dot{x}_{1}\right)=a\left(\left(b x_{1}+c x_{2}-x_{1} x_{3}+u_{2}\right)-a\left(x_{2}\right.\right. \\
& \left.\left.-x_{1}\right)\right) \\
\dot{x}_{1} & +x_{1}=a\left(\dot{x}_{2}-\dot{x}_{1}\right)-\ddot{x}_{1}+\dot{x}_{1}+x_{1}=a\left(b x_{1}+c x_{2}\right. \\
& \left.-x_{1} x_{3}+u_{2}\right)+a(1-a)\left(x_{2}-x_{1}\right)-\ddot{x}_{1}+x_{1} \\
& =a\left(b x_{1}+c x_{2}-x_{1} x_{3}\right)+a(1-a)\left(x_{2}-x_{1}\right)-\ddot{x}_{1} \\
& +x_{1}+a u_{2} \\
2 x_{1} & \left(\dot{x}_{1}+x_{1}\right)=2 x_{1}\left(a\left(b x_{1}+c x_{2}-x_{1} x_{3}\right)+a(1-a)\right. \\
& \left.+\left(x_{2}-x_{1}\right)-\ddot{x}_{1}+x_{1}+a u_{2}\right)=2 x_{1}\left(a \left(b x_{1}+c x_{2}\right.\right. \\
& \left.-x_{1} x_{3}\right)+a(1-a)\left(x_{2}-x_{1}\right)-\ddot{x}_{1}+x_{1}-\operatorname{sgn}\left(x_{1}\right) \\
& +M)=2 x_{1} \operatorname{sgn}\left(x_{1}\right)\left(\operatorname { s g n } ( x _ { 1 } ) \left(a\left(b x_{1}+c x_{2}-x_{1} x_{3}\right)\right.\right. \\
& \left.\left.+a(1-a)\left(x_{2}-x_{1}\right)-\ddot{x}_{1}+x_{1}\right)-M\right) \leq 0 .
\end{aligned}
$$

That is,

$$
\dot{x}_{1}^{2}+2 x_{1}^{2} \leq 0, \quad \text { for } t \in\left[t_{2 m}, t_{2 m+1}\right), m=0,1,2, \ldots
$$

Therefore, one gets

$$
x_{1}^{2} \leq e^{-2\left(t-t_{2 m}\right)} x_{1}^{2}\left(t_{2 m}\right)
$$

Thus, we have

$$
\left|x_{1}\right| \leq e^{-\left(t-t_{2 m}\right)}\left|x_{1}\left(t_{2 m}\right)\right| \text {. }
$$

From inequality (7), it is easy to see that if $\alpha_{\max }=\infty$, then $\left|x_{1}\right| \rightarrow 0$ as $t \rightarrow+\infty$. Therefore we have $\lim _{t \rightarrow+\infty} x_{1}=$ $\lim _{t \rightarrow+\infty} x_{2}=\lim _{t \rightarrow+\infty} x_{3}=0$ which means that conclusion (1) of Theorem 4 is correct.

Case 2. If $t \in\left[t_{2 m+1}, t_{2 m+2}\right), m=0,1,2, \ldots$, then $k_{2}(t)=1$ and $k_{1}(t)=0$. From the first equation of system (2), we have

$$
\begin{aligned}
\dot{x}_{1} & =a\left(x_{2}-x_{1}\right)+u_{1}=a\left(x_{2}-x_{1}\right)-a k_{2}(t) x_{2} \\
& =-a x_{1} .
\end{aligned}
$$

That is

$$
\dot{x}_{1}=-a x_{1} \quad \text { for } t \in\left[t_{2 m+1}, t_{2 m+2}\right), m=0,1,2, \ldots .
$$

Therefore, one gets

$$
\left|x_{1}\right|=e^{-a\left(t-t_{2 m+1}\right)}\left|x_{1}\left(t_{2 m+1}\right)\right| \text {. }
$$

From inequality (10), it is obvious that if $\beta_{\max }=\infty$ and $a>0$, then $\left|x_{1}\right| \rightarrow 0$ as $t \rightarrow+\infty$. Therefore we obtain $\lim _{t \rightarrow+\infty} x_{1}=\lim _{t \rightarrow+\infty} x_{2}=\lim _{t \rightarrow+\infty} x_{3}=0$ which implies that conclusion (2) of Theorem 4 is correct.

Now, we assume that $\alpha_{\max } \beta_{\max } \neq \infty, a<0$.

For $t \in\left[t_{0}, t_{1}\right)$, based on inequality (7), one gets

$$
\left|x_{1}(t)\right| \leq e^{-t}\left|x_{1}(0)\right|
$$

which leads to

$$
\left|x_{1}\left(t_{1}\right)\right| \leq e^{-\alpha_{1}}\left|x_{1}(0)\right| \text {. }
$$

For $t \in\left[t_{1}, t_{2}\right)$, based on inequality (10), we have

$$
\left|x_{1}(t)\right|=e^{-a\left(t-t_{1}\right)}\left|x_{1}\left(t_{1}\right)\right|,
$$

which implies

$$
\left|x_{1}\left(t_{2}\right)\right| \leq e^{-\alpha_{1}-a \beta_{1}}\left|x_{1}(0)\right| .
$$

In general, for $t \in\left[t_{2 m}, t_{2 m+1}\right)$ one obtains that

$$
\begin{aligned}
& \left|x_{1}(t)\right| \\
& \quad \leq e^{-\left(\alpha_{1}+\alpha_{2}+\cdots+\alpha_{m-1}\right)} e^{-a\left(\beta_{1}+\beta_{2}+\cdots+\beta_{m-1}\right)} e^{-\left(t-t_{2 m}\right)}\left|x_{1}(0)\right| \\
& \quad \leq e^{-(m-1) \alpha_{\min }} e^{-a(m-1) \beta_{\max }} e^{-\left(t-t_{2 m}\right)}\left|x_{1}(0)\right| \\
& \quad=e^{-(m-1)\left(\alpha_{\min }+a \beta_{\max }\right)} e^{-\left(t-t_{2 m}\right)}\left|x_{1}(0)\right| \\
& \quad \leq e^{-(m-1)\left(\alpha_{\min }+a \beta_{\max }\right)}\left|x_{1}(0)\right| .
\end{aligned}
$$

Since $\alpha_{\max } \beta_{\max } \neq \infty$, it is easy to see that $t \rightarrow \infty$ implies that $m \rightarrow \infty$. Note that $\alpha_{\min }+a \beta_{\max } \geq \varepsilon>0$; thus we have $-(m-1)\left(\alpha_{\min }+a \beta_{\max }\right) \rightarrow-\infty$ as $t \rightarrow+\infty$ which implies that $\lim _{t \rightarrow+\infty} x_{1}=\lim _{t \rightarrow+\infty} x_{2}=\lim _{t \rightarrow+\infty} x_{3}=0$.

For $t \in\left[t_{2 m+1}, t_{2 m+2}\right)$ one gets that

$$
\begin{aligned}
& \left|x_{1}(t)\right| \\
& \quad \leq e^{-\left(\alpha_{1}+\alpha_{2}+\cdots+\alpha_{m}\right)} e^{-a\left(\beta_{1}+\beta_{2}+\cdots+\beta_{m}\right)} e^{-a\left(t-t_{2 m+1}\right)}\left|x_{1}(0)\right| .
\end{aligned}
$$

Note that $a<0$, and we obtain that

$$
\left|x_{1}(t)\right| \leq e^{-m\left(\alpha_{\min }+a \beta_{\max }\right)} e^{-a \beta_{\max }}\left|x_{1}(0)\right| .
$$

Since $m \rightarrow \infty$ and $\alpha_{\min }+a \beta_{\max } \geq \varepsilon>0$, we have $-m\left(\alpha_{\min }+a \beta_{\max }\right) \rightarrow-\infty$ as $t \rightarrow+\infty$ which implies that $\lim _{t \rightarrow+\infty} x_{1}=\lim _{t \rightarrow+\infty} x_{2}=\lim _{t \rightarrow+\infty} x_{3}=0$. Therefore, conclusion (3) of Theorem 4 is correct.

In the following, we assume that $\alpha_{\max } \beta_{\max } \neq \infty, a>0$.

For $t \in\left[t_{2 m}, t_{2 m+1}\right)$, based on inequality (15), one gets

$$
\begin{aligned}
& \left|x_{1}(t)\right| \\
& \quad \leq e^{-\left(\alpha_{1}+\alpha_{2}+\cdots+\alpha_{m-1}\right)} e^{-a\left(\beta_{1}+\beta_{2}+\cdots+\beta_{m-1}\right)} e^{-\left(t-t_{2 m}\right)}\left|x_{1}(0)\right| \\
& \quad \leq e^{-(m-1) \alpha_{\min }}\left|x_{1}(0)\right| .
\end{aligned}
$$

Note that in this case $\alpha_{\min }>0$ and $m \rightarrow+\infty$ as $t \rightarrow+\infty$; we know that $\left|x_{1}(t)\right| \rightarrow 0$ as $t \rightarrow+\infty$ which implies that $\lim _{t \rightarrow+\infty} x_{1}=\lim _{t \rightarrow+\infty} x_{2}=\lim _{t \rightarrow+\infty} x_{3}=0$. 
For $t \in\left[t_{2 m+1}, t_{2 m+2}\right)$, based on inequality (16), one gets

$$
\left|x_{1}(t)\right| \leq e^{-m \alpha_{\min }}\left|x_{1}(0)\right|
$$

Note that in this case $\alpha_{\min }>0$ and $m \rightarrow+\infty$ as $t \rightarrow$ $+\infty$; we obtain that $\left|x_{1}(t)\right| \rightarrow 0$ as $t \rightarrow+\infty$ which means that $\lim _{t \rightarrow+\infty} x_{1}=\lim _{t \rightarrow+\infty} x_{2}=\lim _{t \rightarrow+\infty} x_{3}=0$. Therefore, conclusion (4) of Theorem 4 is correct. This completes the proof of Theorem 4 .

Remark 5. It is well known that the states of chaotic systems are bounded which means that $x_{1}, x_{2}$, and $x_{3}$ are all bounded.

In order to prove $\mid a\left(b x_{1}+c x_{2}-x_{1} x_{3}\right)+a(1-a)\left(x_{2}-x_{1}\right)-$ $\ddot{x}_{1}+x_{1} \mid \leq M$ we only need to show that $\ddot{x}_{1}$ is bounded. In the following a simple proof shows that $\ddot{x}_{1}$ is bounded.

From system (2) and Theorem 4 we know

$$
\begin{aligned}
& \dot{x}_{1}=a\left(x_{2}-x_{1}\right)+u_{1}, \\
& \dot{x}_{2}=b x_{1}+c x_{2}-x_{1} x_{3}+u_{2},
\end{aligned}
$$

where

$$
\begin{aligned}
& u_{1}=-a k_{2}(t)\left(k_{1}(t) x_{1}+k_{2}(t) x_{2}\right), \\
& u_{2}=-k_{1}(t) \operatorname{sgn}\left(k_{1}(t) x_{1}+k_{2}(t) x_{2}\right) \frac{M}{a} .
\end{aligned}
$$

Since $x_{1}, x_{2}$, and $x_{3}$ are all bounded, based on (20)(21), one can easily derive that $\dot{x}_{1}, \dot{x}_{2}$, and $u_{2}$ are bounded. Furthermore, by (21) we can obtain that $\dot{u}_{1}$ is bounded.

Now, in view of

$$
\begin{aligned}
\ddot{x}_{1} & =a\left(\dot{x}_{2}-\dot{x}_{1}\right)+\dot{u}_{1} \\
& =a\left(\left(b x_{1}+c x_{2}-x_{1} x_{3}+u_{2}\right)-a\left(x_{2}-x_{1}\right)\right)+\dot{u}_{1}
\end{aligned}
$$

we conclude that $\ddot{x}_{1}$ is bounded which implies that Assumption 3 is reasonable.

\section{The Synchronization of a Class of Chaotic Systems}

Suppose system (1) is the drive system. In order to synchronize system (1), we introduce the following response system:

$$
\begin{aligned}
\hat{x}_{1}= & k_{1}(t) w_{11}+k_{1}(t) x_{\text {out }}+k_{2}(t) w_{21} \\
& +\frac{k_{2}(t)\left(l_{21}-a\right)}{b} x_{\text {out }}, \\
\dot{w}_{11}= & -l_{11}\left(\widehat{x}_{1}-x_{\text {out }}\right), \\
\dot{w}_{21}= & -l_{21} \widehat{x}_{1}-\frac{\left(l_{21}-a\right) c-a b}{b} \widehat{x}_{2}+\frac{l_{21}-a}{b} \widehat{x}_{1} \widehat{x}_{3}, \\
\hat{x}_{2}= & k_{1}(t) w_{12}+k_{1}(t) \frac{l_{12}+c}{a} x_{\text {out }}+k_{2}(t) w_{22} \\
& +k_{2}(t) \frac{l_{22}+c}{c} x_{\text {out }}, \\
\dot{w}_{12}= & -l_{12} w_{12}-\frac{l_{12}\left(l_{12}+c\right)}{a} x_{\text {out }}+\left(l_{12}+c\right) x_{\text {out }} \\
& +b \widehat{x}_{1}-\widehat{x}_{1} \widehat{x}_{3}, \\
\dot{w}_{22}= & -\frac{l_{22} b}{c} \widehat{x}_{1}-l_{22} \widehat{x}_{2}+\frac{l_{22}}{c} \widehat{x}_{1} \widehat{x}_{3}, \\
\dot{\hat{x}}_{3}= & \widehat{x}_{1} \hat{x}_{2}-d \widehat{x}_{3},
\end{aligned}
$$

where $\hat{x}_{1}, \hat{x}_{2}$, and $\hat{x}_{3}$ are the estimated values of $x_{1}, x_{2}$, and $x_{3}$, respectively. $w_{i j}, i, j=1,2$, are intermediate variables. $l_{i 1}$ and $l_{i 2}, i=1,2$, are positive numbers to be designed later.

Prior to going any further, we make the following assumption.

Assumption 6. Suppose the state variables of systems (1) and (23) are bounded, which means that there exists nonnegative constant $M$ such that $\left|x_{i}\right| \leq M,\left|\widehat{x}_{i}\right| \leq M, i=1,2,3$.

Let $\lambda_{1}, \lambda_{2}$ denote the minimum eigenvalues of matrix $Q_{1}$, $Q_{2}$, respectively, where

$$
\begin{aligned}
& Q_{1}=\left(\begin{array}{ccc}
l_{1} & -\frac{1}{2}(|b|+M) & -\frac{1}{2} M \\
-\frac{1}{2}(|b|+M) & l_{2} & 0 \\
-\frac{1}{2} M & 0 & d
\end{array}\right), \\
& Q_{2}=\left(\begin{array}{ccc}
-\frac{1}{2}\left(\left|\frac{\left(l_{21}-a\right) c-a b}{b}+\frac{l_{22} b}{c}\right|+\left|\frac{l_{22}}{c}\right| M\right) & -\frac{1}{2}\left(\left|\frac{\left(l_{21}-a\right) c-a b}{b}+\frac{l_{22} b}{c}\right|+\left|\frac{l_{22}}{c}\right| M\right)-\frac{1}{2}\left(\left|\frac{l_{21-a}}{b}\right|+1\right) M \\
l_{21}-\left|\frac{l_{21}-a}{b}\right| M & -\frac{1}{2}\left(\left|\frac{l_{22}}{c}\right|+1\right) M \\
-\frac{1}{2}\left(\left|\frac{l_{21}-a}{b}\right|+1\right) M & -\frac{1}{2}\left(\left|\frac{l_{22}}{c}\right|+1\right) M
\end{array}\right) .
\end{aligned}
$$


Then we have the following theorem.

Theorem 7. (1) If $\alpha_{\max }=\infty$ and there exist nonnegative constants $l_{11}$ and $l_{12}$ such that $Q_{1}>0$, then system (23) can synchronize system (1) in the sense of $\lim _{t \rightarrow+\infty} e_{1}=$ $\lim _{t \rightarrow+\infty} e_{2}=\lim _{t \rightarrow+\infty} e_{3}=0$.

(2) If $\beta_{\max }=\infty$ and there exist nonnegative constants $l_{21}$ and $l_{22}$ such that $Q_{2}>0$, then system (23) can synchronize system (1) in the sense of $\lim _{t \rightarrow+\infty} e_{1}=\lim _{t \rightarrow+\infty} e_{2}=$ $\lim _{t \rightarrow+\infty} e_{3}=0$.

(3) For $\alpha_{\max } \beta_{\max } \neq \infty$.

(a) If $\lambda_{1}>0$ and there exists constant $\varepsilon>0$ such that $\lambda_{1} \alpha_{\min }+\lambda_{2} \beta_{\max } \geq \varepsilon$, then system (23) can synchronize system (1) in the sense of $\lim _{t \rightarrow+\infty} e_{1}=\lim _{t \rightarrow+\infty} e_{2}=\lim _{t \rightarrow+\infty} e_{3}=0$.

(b) If $\lambda_{2}>0$ and there exists constant $\varepsilon>0$ such that $\lambda_{2} \beta_{\min }+\lambda_{1} \alpha_{\max } \geq \varepsilon$, then system (23) can synchronize system (1) in the sense of $\lim _{t \rightarrow+\infty} e_{1}=\lim _{t \rightarrow+\infty} e_{2}=\lim _{t \rightarrow+\infty} e_{3}=0$, where $e_{1}=\widehat{x}_{1}-x_{1}, e_{2}=\widehat{x}_{2}-x_{2}$, and $e_{3}=\widehat{x}_{3}-x_{3} . \lambda_{1}$ and $\lambda_{2}$ are the minimum eigenvalues of matrixes $Q_{1}$ and $Q_{2}$, respectively.

Proof. Let $e_{1}=\widehat{x}_{1}-x_{1}, e_{2}=\widehat{x}_{2}-x_{2}$, and $e_{3}=\widehat{x}_{3}-x_{3}$. Two cases are discussed in the following.

Case 1. If $t \in\left[t_{2 m}, t_{2 m+1}\right), m=0,1,2, \ldots$, then $k_{1}(t)=1$, $k_{2}(t)=0$, and $x_{\text {out }}=x_{1}$. In this case from systems (1) and (23) we have

$$
\begin{aligned}
& \dot{e}_{1}=\dot{\bar{x}}_{1}-\dot{x}_{1} \\
& =-l_{11}\left(\hat{x}_{1}-x_{\text {out }}\right)+a\left(x_{2}-x_{1}\right)-a\left(x_{2}-x_{1}\right) \\
& =-l_{11} e_{1} \\
& \dot{\hat{x}}_{2}=\dot{w}_{12}+\frac{l_{12}+c}{a} \dot{x}_{\text {out }} \\
& =-l_{12} w_{12}-\frac{l_{12}\left(l_{12}+c\right)}{a} x_{\text {out }}+\left(l_{12}+c\right) x_{\text {out }}+b \widehat{x}_{1} \\
& -\widehat{x}_{1} \widehat{x}_{3}+\frac{l_{12}+c}{a} a\left(x_{2}-x_{1}\right) \\
& =-l_{12}\left(\hat{x}_{2}-\frac{l_{12}+c}{a} x_{\text {out }}\right)-\frac{l_{12}\left(l_{12}+c\right)}{a} x_{\text {out }} \\
& +\left(l_{12}+c\right) x_{\text {out }}+b \widehat{x}_{1}-\widehat{x}_{1} \widehat{x}_{3} \\
& +\frac{l_{12}+c}{a} a\left(x_{2}-x_{1}\right) \\
& =-l_{12} \widehat{x}_{2}+\left(l_{12}+c\right) x_{\text {out }}+b \widehat{x}_{1}-\widehat{x}_{1} \widehat{x}_{3} \\
& +\left(l_{12}+c\right)\left(x_{2}-x_{1}\right) \\
& =-l_{12} \widehat{x}_{2}+b \hat{x}_{1}-\hat{x}_{1} \hat{x}_{3}+\left(l_{12}+c\right) x_{2}, \\
& \dot{e}_{2}=-l_{12} \widehat{x}_{2}+b \widehat{x}_{1}-\hat{x}_{1} \hat{x}_{3}+\left(l_{12}+c\right) x_{2}-b x_{1}-c x_{2} \\
& +x_{1} x_{3}=-l_{12} e_{2}+b e_{1}-x_{1} e_{3}-\hat{x}_{3} e_{1} \\
& =-l_{12} e_{2}+\left(b-\widehat{x}_{3}\right) e_{1}-x_{1} e_{3} \text {, } \\
& \dot{e}_{3 x}=\hat{x}_{1} \hat{x}_{2}-d \hat{x}_{3}-\left(x_{1} x_{2}-d x_{3}\right) \\
& =x_{1} e_{2}+\widehat{x}_{2} e_{1}-d e_{3} \text {. }
\end{aligned}
$$

Thus, we get the error dynamical system:

$$
\begin{aligned}
& \dot{e}_{1}=-l_{11} e_{1}, \\
& \dot{e}_{2}=-l_{12} e_{2}+\left(b-\hat{x}_{3}\right) e_{1}-x_{1} e_{3}, \\
& \dot{e}_{3}=x_{1} e_{2}+\widehat{x}_{2} e_{1}-d e_{3} .
\end{aligned}
$$

For system (26), if we take Lyapunov candidate

$$
V=\frac{1}{2}\left(e_{1}^{2}+e_{2}^{2}+e_{3}^{2}\right),
$$

then the derivative of (27) is

$$
\begin{aligned}
\dot{V}= & e_{1}\left(-l_{11} e_{1}\right)+e_{2}\left(-l_{12} e_{2}+\left(b-\widehat{x}_{3}\right) e_{1}-x_{1} e_{3}\right) \\
& +e_{3}\left(x_{1} e_{2}+\widehat{x}_{2} e_{1}-d e_{3}\right) \\
= & -l_{11} e_{1}^{2}-l_{12} e_{2}^{2}+\left(b-\widehat{x}_{3}\right) e_{1} e_{2}-x_{1} e_{3} e_{2}+x_{1} e_{2} e_{3} \\
& +\widehat{x}_{2} e_{1} e_{3}-d e_{3}^{2} \\
\leq & -l_{11} e_{1}^{2}-l_{12} e_{2}^{2}+(|b|+M)\left|e_{1} e_{2}\right|+M\left|e_{1} e_{3}\right|-d e_{3}^{2} \\
= & -\left(\left|e_{1 x}\right|,\left|e_{2 x}\right|,\left|e_{3 x}\right|\right) Q_{1}\left(\left|e_{1 x}\right|,\left|e_{2 x}\right|,\left|e_{3 x}\right|\right)^{T} \\
\leq & -2 \lambda_{1} V .
\end{aligned}
$$

Thus, we have

$$
|V(t)| \leq e^{-2 \lambda_{1}\left(t-t_{2 m}\right)}\left|V\left(t_{2 m}\right)\right| .
$$

From inequality (29), it is easy to see that if $\alpha_{\max }=\infty$, then $|V| \rightarrow 0$ as $t \rightarrow+\infty$. Therefore we have $\lim _{t \rightarrow+\infty} e_{1}=$ $\lim _{t \rightarrow+\infty} e_{2}=\lim _{t \rightarrow+\infty} e_{3}=0$ which means that conclusion (1) of Theorem 7 is correct.

Case 2. If $t \in\left[t_{2 m+1}, t_{2 m+2}\right), m=0,1,2, \ldots$, then $k_{2}(t)=1$, $k_{1}(t)=0$, and $x_{\text {out }}=x_{2}$. In this case from systems (1) and (23) we have

$$
\begin{aligned}
\dot{e}_{1}= & -l_{21} \widehat{x}_{1}-\frac{\left(l_{21}-a\right) c-a b}{b} \widehat{x}_{2}+\frac{l_{21}-a}{b} \widehat{x}_{1} \widehat{x}_{3} \\
& +\left(l_{21}-a\right) x_{1}+\frac{\left(l_{21}-a\right) c}{b} x_{2}-\frac{l_{21}-a}{b} x_{1} x_{3} \\
& -a x_{2}+a x_{1} \\
= & -l_{21} \widehat{x}_{1}-\frac{\left(l_{21}-a\right) c-a b}{b} \widehat{x}_{2}+\frac{l_{21}-a}{b} \widehat{x}_{1} \widehat{x}_{3} \\
& +l_{21} x_{1}+\frac{\left(l_{21}-a\right) c}{b} x_{2}-\frac{l_{21}-a}{b} x_{1} x_{3}-a x_{2} \\
= & -l_{21} e_{1}-\frac{\left(l_{21}-a\right) c-a b}{b} e_{2} \\
& +\frac{l_{21}-a}{b}\left(\widehat{x}_{1} \widehat{x}_{3}-x_{1} x_{3}\right) .
\end{aligned}
$$




$$
\begin{aligned}
\dot{e}_{2}= & \dot{w}_{22}+\frac{l_{22}+c}{c}\left(b x_{1}+c x_{2}-x_{1} x_{3}\right) \\
& -\left(b x_{1}+c x_{2}-x_{1} x_{3}\right) \\
= & -\frac{l_{22} b}{c} \hat{x}_{1}-l_{22} \hat{x}_{2}+\frac{l_{22}}{c} \hat{x}_{1} \hat{x}_{3}+\frac{l_{22} b}{c} x_{1}+l_{22} x_{2} \\
& -\frac{l_{22}}{c} x_{1} x_{3} \\
= & -\frac{l_{22} b}{c} e_{1}-l_{22} e_{2}+\frac{l_{22}}{c}\left(\hat{x}_{1} \hat{x}_{3}-x_{1} x_{3}\right) . \\
\dot{e}_{3}= & \left(\hat{x}_{1} \hat{x}_{2}-x_{1} x_{2}\right)-d e_{3} .
\end{aligned}
$$

Thus, we get the error dynamical system:

$$
\begin{aligned}
\dot{e}_{1}= & -l_{21} e_{1}-\frac{\left(l_{21}-a\right) c-a b}{b} e_{2} \\
& +\frac{l_{21}-a}{b}\left(\hat{x}_{1} \hat{x}_{3}-x_{1} x_{3}\right), \\
\dot{e}_{2}= & -\frac{l_{22} b}{c} e_{1}-l_{22} e_{2}+\frac{l_{22}}{c}\left(\hat{x}_{1} \hat{x}_{3}-x_{1} x_{3}\right), \\
\dot{e}_{3}= & \left(\hat{x}_{1} \hat{x}_{2}-x_{1} x_{2}\right)-d e_{3} .
\end{aligned}
$$

For system (31), if we take Lyapunov candidate

$$
V=\frac{1}{2}\left(e_{1}^{2}+e_{2}^{2}+e_{3}^{2}\right)
$$

then the derivative of (32) is

$$
\begin{aligned}
\dot{V} & =e_{1}\left(-l_{21} e_{1}-\frac{\left(l_{21}-a\right) c-a b}{b} e_{2}\right. \\
& \left.+\frac{l_{21}-a}{b}\left(\hat{x}_{1} \hat{x}_{3}-x_{1} x_{3}\right)\right)+e_{2}\left(-\frac{l_{22} b}{c} e_{1}-l_{22} e_{2}\right. \\
& \left.+\frac{l_{22}}{c}\left(\hat{x}_{1} \hat{x}_{3}-x_{1} x_{3}\right)\right)+e_{3}\left(\hat{x}_{1} \hat{x}_{2}-x_{1} x_{2}-d e_{3}\right) \\
& =-l_{21} e_{1}^{2}-\frac{\left(l_{21}-a\right) c-a b}{b} e_{1} e_{2}+\frac{l_{21}-a}{b} x_{3} e_{1}^{2} \\
& +\frac{l_{21}-a}{b} \hat{x}_{1} e_{1} e_{3}-\frac{l_{22} b}{c} e_{1} e_{2}-l_{22} e_{2}^{2}+\frac{l_{22}}{c} \hat{x}_{1} e_{2} e_{3} \\
& +\frac{l_{22}}{c} x_{3} e_{1} e_{2}+\hat{x}_{1} e_{2} e_{3}+x_{2} e_{1} e_{3}-d e_{3}^{2}=-\left(l_{21}\right. \\
& \left.-\frac{l_{21}-a}{b} x_{3}\right) e_{1}^{2}-\left(\frac{\left(l_{21}-a\right) c-a b}{b}+\frac{l_{22} b}{c}\right. \\
& \left.-\frac{l_{22}}{c} x_{3}\right) e_{1} e_{2}+\left(\frac{l_{21}-a}{b} \hat{x}_{1}+x_{2}\right) e_{1} e_{3}-l_{22} e_{2}^{2}
\end{aligned}
$$

$$
\begin{aligned}
& +\left(\frac{l_{22}}{c} \hat{x}_{1}+\widehat{x}_{1}\right) e_{2} e_{3}-d e_{3}^{2} \leq-\left(l_{21}-\left|\frac{l_{21}-a}{b}\right| M\right) \\
& \cdot e_{1}^{2}+\left(\left|\frac{\left(l_{21}-a\right) c-a b}{b}+\frac{l_{22} b}{c}\right|+\left|\frac{l_{22}}{c}\right| M\right)\left|e_{1} e_{2}\right| \\
& +\left(\left|\frac{l_{21}-a}{b}\right|+1\right) M\left|e_{1} e_{3}\right|-l_{22} e_{2}^{2}+\left(\left|\frac{l_{22}}{c}\right|+1\right) \\
& \cdot M\left|e_{2} e_{3}\right|-d e_{3}^{2}=-\left(\left|e_{1}\right|,\left|e_{2}\right|,\left|e_{3}\right|\right) \\
& \cdot Q_{2}\left(\left|e_{1}\right|,\left|e_{2}\right|,\left|e_{3}\right|\right)^{T} \leq-2 \lambda_{2} V .
\end{aligned}
$$

Thus, we have

$$
|V(t)| \leq e^{-2 \lambda_{2}\left(t-t_{2 m+1}\right)}\left|V\left(t_{2 m+1}\right)\right| .
$$

From inequality (34), it is easy to see that if $\beta_{\max }=\infty$, then $|V| \rightarrow 0$ as $t \rightarrow+\infty$. Therefore we have $\lim _{t \rightarrow+\infty} e_{1}=$ $\lim _{t \rightarrow+\infty} e_{2}=\lim _{t \rightarrow+\infty} e_{3}=0$ which means that conclusion (2) of Theorem 7 is correct.

In the following, we assume that $\alpha_{\max } \beta_{\max } \neq \infty$. Suppose $\lambda_{1}>0$.

For $t \in\left[t_{0}, t_{1}\right)$, based on inequality (29) one gets

$$
V(t) \leq e^{-2 \lambda_{1} t} V(0),
$$

which leads to

$$
V\left(t_{1}\right) \leq e^{-2 \lambda_{1} \alpha_{1}} V(0) .
$$

For $t \in\left[t_{1}, t_{2}\right)$, based on inequality (34) we have

$$
|V(t)| \leq e^{-2 \lambda_{2}\left(t-t_{1}\right)}\left|V\left(t_{1}\right)\right|,
$$

which implies

$$
\left|V\left(t_{2}\right)\right| \leq e^{-2 \lambda_{2} \beta_{1}}\left|V\left(t_{1}\right)\right|
$$

In general, for $t \in\left[t_{2 m}, t_{2 m+1}\right)$ one obtains that

$|V(t)|$

$$
\begin{aligned}
& \leq e^{-2 \lambda_{1}\left(\alpha_{1}+\alpha_{2}+\cdots+\alpha_{m-1}\right)} e^{-2 \lambda_{2}\left(\beta_{1}+\beta_{2}+\cdots+\beta_{m-1}\right)} e^{-\left(t-t_{2 m}\right)}|V(0)| \\
& \leq e^{-2 \lambda_{1}(m-1) \alpha_{\min }} e^{-2 \lambda_{2}(m-1) \beta_{\max }} e^{-\left(t-t_{2 m}\right)}|V(0)| \\
& =e^{-2(m-1)\left(\lambda_{1} \alpha_{\min }+\lambda_{2} \beta_{\max }\right)} e^{-\left(t-t_{2 m}\right)}|V(0)| \\
& \leq e^{-2(m-1)\left(\lambda_{1} \alpha_{\min }+\lambda_{2} \beta_{\max }\right)}|V(0)| .
\end{aligned}
$$

Since $\alpha_{\max } \beta_{\max } \neq \infty$, it is easy to see that $t \rightarrow \infty$ implies that $m \rightarrow \infty$. Note that $\lambda_{1} \alpha_{\text {min }}+\lambda_{2} \beta_{\max } \geq \varepsilon>0$; thus we have $-(m-1)\left(\lambda_{1} \alpha_{\min }+\lambda_{2} \beta_{\max }\right) \rightarrow-\infty$ as $t \rightarrow+\infty$ which implies that $\lim _{t \rightarrow+\infty} e_{1}=\lim _{t \rightarrow+\infty} e_{2}=\lim _{t \rightarrow+\infty} e_{3}=0$.

For $t \in\left[t_{2 m+1}, t_{2 m+2}\right)$ one gets that

$|V(t)|$

$$
\leq e^{-2 \lambda_{1}\left(\alpha_{1}+\alpha_{2}+\cdots+\alpha_{m}\right)} e^{-2 \lambda_{2}\left(\beta_{1}+\beta_{2}+\cdots+\beta_{m}\right)} e^{-2 \lambda_{2}\left(t-t_{2 m+1}\right)}|V(0)| .
$$


If $\lambda_{2} \leq 0$, from the inequality (40), we obtain

$$
|V(t)| \leq e^{-2 m\left(\lambda_{1} \alpha_{\min }+\lambda_{2} \beta_{\max }\right)} e^{-2 \lambda_{2} \beta_{\max }}|V(0)| .
$$

Since $m \rightarrow \infty$ and $\lambda_{1} \alpha_{\min }+\lambda_{2} \beta_{\max } \geq \varepsilon>0$, we have $-2 m\left(\lambda_{1} \alpha_{\min }+\lambda_{2} \beta_{\max }\right) \rightarrow-\infty$ as $t \rightarrow+\infty$ which implies that $\lim _{t \rightarrow+\infty} e^{-2 m\left(\lambda_{1} \alpha_{\min }+\lambda_{2} \beta_{\max }\right)}=0$. Note that $e^{-2 \lambda_{2} \beta_{\max }}|V(0)|$ is a constant; we derive $\lim _{t \rightarrow+\infty} e^{-2 m\left(\lambda_{1} \alpha_{\min }+\lambda_{2} \beta_{\max }\right)} e^{-2 \lambda_{2} \beta_{\max }}|V(0)|=0$ which means that $\lim _{t \rightarrow+\infty}|V(t)|=0$. In view of $V=(1 / 2)\left(e_{1}^{2}+e_{2}^{2}+e_{3}^{2}\right)$ we have $\lim _{t \rightarrow+\infty} e_{1}=\lim _{t \rightarrow+\infty} e_{2}=\lim _{t \rightarrow+\infty} e_{3}=0$.

If $\lambda_{2}>0$, from the inequality (40), we get

$$
|V(t)| \leq e^{-2 m\left(\lambda_{1} \alpha_{\min }+\lambda_{2} \beta_{\max }\right)}|V(0)| .
$$

Along the same lines, one gets $\lim _{t \rightarrow+\infty} e_{1}=\lim _{t \rightarrow+\infty} e_{2}=$ $\lim _{t \rightarrow+\infty} e_{3}=0$. Therefore, conclusion (3) (a) of Theorem 7 is correct. In the same way we can prove that conclusion (3) (b) of Theorem 7 is correct. This completes the proof of Theorem 7.

$$
\begin{aligned}
|V(t)| & \leq e^{-2 m\left(\lambda_{1} \alpha_{\min }+\lambda_{2} \beta_{\max }\right)} e^{-2 \lambda_{2} \beta_{\max }}|V(0)| \\
& \leq e^{-2 m\left(\lambda_{1} \alpha_{\min }+\lambda_{2} \beta_{\max }\right)}|V(0)| .
\end{aligned}
$$

Since $m \rightarrow \infty$ and $\lambda_{1} \alpha_{\min }+\lambda_{2} \beta_{\max } \geq \varepsilon>0$, we have $-2 m\left(\lambda_{1} \alpha_{\min }+\lambda_{2} \beta_{\max }\right) \rightarrow-\infty$ as $t \rightarrow+\infty$ which implies that $\lim _{t \rightarrow+\infty} e_{1}=\lim _{t \rightarrow+\infty} e_{2}=\lim _{t \rightarrow+\infty} e_{3}=0$. Therefore, conclusion (3) (a) of Theorem 7 is correct. In the same way we can proof that conclusion (3) (b) of Theorem 7 is correct. This completes the proof of Theorem 7.

Remark 8. It is easy to see that in our synchronization scheme we do not need to restrict $c<0$.

\section{Numerical Simulations}

In this section, we will take the generalized Lorenz chaotic system as an example to illustrate the effectiveness of the proposed methods. The generalized Lorenz chaotic system is given by the following set of differential equations [17]:

$$
\begin{aligned}
& \dot{x}_{1}=\left(10+\frac{25}{29} \mu\right)\left(x_{2}-x_{1}\right), \\
& \dot{x}_{2}=\left(28-\frac{35}{29} \mu\right) x_{1}+(\mu-1) x_{2}-x_{1} x_{3}, \\
& \dot{x}_{3}=\left(-\frac{8}{3}-\frac{1}{87} \mu\right) x_{3}+x_{1} x_{2},
\end{aligned}
$$

where $x=\left(x_{1}, x_{2}, x_{3}\right)^{T} \in R^{3 \times 1}$ is the state vector of system (44), and the system parameter $\mu$ satisfies $\mu \in\{\mu \mid-232<$ $\mu<-11.6\} \cup\{\mu \mid \mu>-11.6\}$. System (44) is chaotic for each $0 \leq \mu \leq 29$. Since in our control and synchronization schemes we need $\mu-1<0$, we set $\mu=1 / 10$. The dynamic behavior with $\mu=1 / 10$ and $\left(x_{1}(0), x_{2}(0), x_{3}(0)\right)=(6,8,10)$ is shown in Figure 1.
System (44) with output variable can be rewritten as

$$
\begin{aligned}
& \dot{x}_{1}=\left(10+\frac{2.5}{29}\right)\left(x_{2}-x_{1}\right), \\
& \dot{x}_{2}=\left(28-\frac{3.5}{29}\right) x_{1}-0.9 x_{2}-x_{1} x_{3}, \\
& \dot{x}_{3}=\left(-\frac{8}{3}-\frac{0.1}{87}\right) x_{3}+x_{1} x_{2}, \\
& x_{\text {out }}=k_{1}(t) x_{1}+k_{2}(t) x_{2}, \\
& k_{1}(t) \quad \\
& \quad= \begin{cases}1, & t \in\left[t_{2 m}, t_{2 m+1}\right), t_{0}=0, m=0,1,2, \ldots, \\
0, & t \in\left[t_{2 m+1}, t_{2 m+2}\right), m=0,1,2, \ldots,\end{cases} \\
& k_{2}(t) \quad= \begin{cases}0, & t \in\left[t_{2 m}, t_{2 m+1}\right), t_{0}=0, m=0,1,2, \ldots, \\
1, & t \in\left[t_{2 m+1}, t_{2 m+2}\right), m=0,1,2, \ldots .\end{cases}
\end{aligned}
$$

By comparing with system (1) it is easy to see that $a=10+$ $2.5 / 29, b=28-3.5 / 29, c=-0.9$, and $d=8 / 3+0.1 / 87$.

Example 9 (the control of the generalized Lorenz chaotic system). In this simulation process, we suppose that $M=$ $100 a$ and the controllers $u_{1}$ and $u_{2}$ are taken as that of Theorem 4 . Then, according to Theorem 4 we have $\lim _{t \rightarrow+\infty} x_{1}=$ $\lim _{t \rightarrow+\infty} x_{2}=\lim _{t \rightarrow+\infty} x_{3}=0$. The simulation result with $x_{1}(0)=3, x_{2}(0)=5$, and $x_{3}(0)=-4$ is shown in Figure 2. It can be seen that the orbits of system (45) approach the origin asymptotically.

In order to show the robust of our control scheme to parameter uncertainties and external disturbances, we add some parameter uncertainties and external disturbances to system (45). Thus the controlled system (45) is rewritten as

$$
\begin{aligned}
\dot{x}_{1}= & \left(10+\frac{2.5}{29}+0.1\right)\left(x_{2}-x_{1}\right)-0.2 x_{1} x_{2}+u_{1}, \\
\dot{x}_{2} & \\
= & \left(28-\frac{3.5}{29}-0.1\right) x_{1}-(0.9-0.1) x_{2}-x_{1} x_{3} \\
& +0.1 x_{2} x_{3}+u_{2}, \\
\dot{x}_{3}= & \left(-\frac{8}{3}-\frac{0.1}{87}-0.1\right) x_{3}+x_{1} x_{2}-0.1 x_{1} x_{3}, \\
x_{\text {out }}= & k_{1}(t) x_{1}+k_{2}(t) x_{2}, \\
k_{1}(t) & \\
=\{ & \begin{cases}1, & t \in\left[t_{2 m}, t_{2 m+1}\right), t_{0}=0, m=0,1,2, \ldots, \\
0, & t \in\left[t_{2 m+1}, t_{2 m+2}\right), m=0,1,2, \ldots,\end{cases}
\end{aligned}
$$




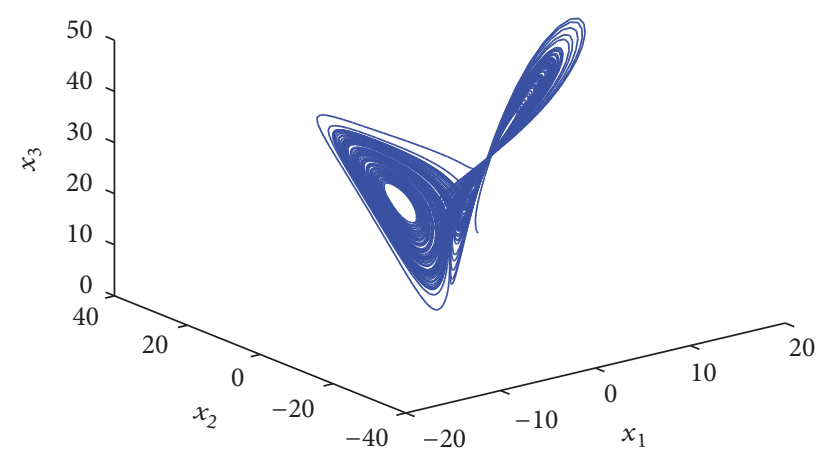

(a)
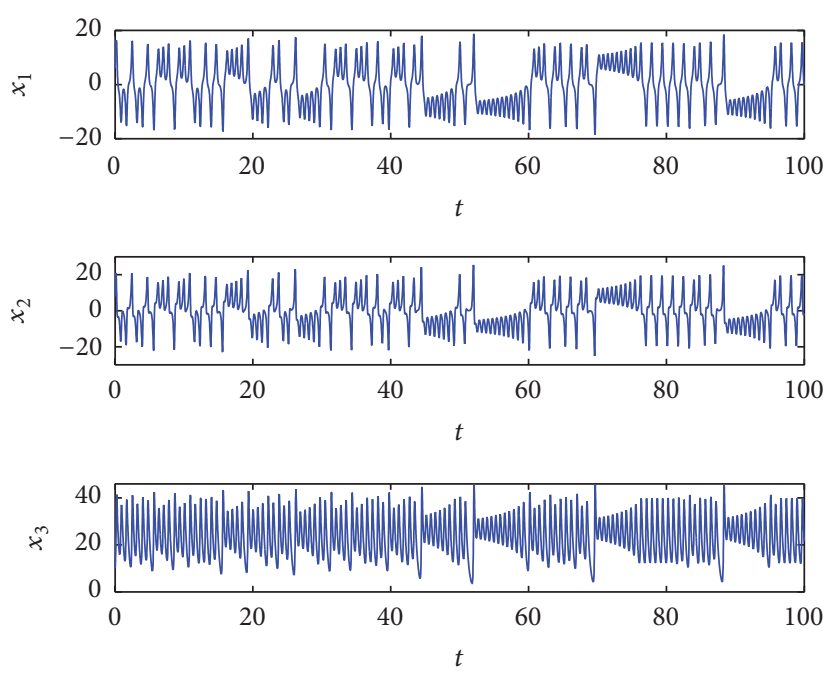

(b)

FIGURE 1: The dynamic behavior of system (44) with $\mu=1 / 10$ and $\left(x_{1}(0), x_{2}(0), x_{3}(0)\right)=(6,8,10)$. (a) The chaotic attractor of system (44). (b) The state trajectories of system (44).

$$
\begin{aligned}
& k_{2}(t) \\
& \quad= \begin{cases}0, & t \in\left[t_{2 m}, t_{2 m+1}\right), t_{0}=0, m=0,1,2, \ldots, \\
1, & t \in\left[t_{2 m+1}, t_{2 m+2}\right), m=0,1,2, \ldots,\end{cases}
\end{aligned}
$$

where $0.1\left(x_{2}-x_{1}\right),-0.1 x_{1}$, and $-0.1 x_{3}$ denote the parameter uncertainties, while $-0.2 x_{1} x_{2}, 0.1 x_{2} x_{3}$, and $-0.1 x_{1} x_{3}$ represent the external disturbances. The simulation result with the same controller and initial conditions as in Figure 2 is given in Figure 3. From Figure 3 one can easily see that the asymptotically stable of the origin of system (46) is actually achieved.

Example 10 (the synchronization scheme of the generalized Lorenz chaotic system). Based on Figure 1, the upper bound $M$ is chosen as $M=45$. If we take $l_{11}=400$ and $l_{12}=100$, then one can easily check that $Q_{1}$ is a positive definite matrix. Its eigenvalues are easy to obtain which are 1.3535, 95.7129, and 405.6014, respectively. Thus we can take $\lambda_{1}=1.3535$. For matrix $Q_{2}$, we suppose that $l_{21}=a=10+2.5 / 29$ and $l_{22}=0$. The eigenvalues of $Q_{2}$ are $-30.8391,9.8910$, and 33.7021, respectively, which means that $\lambda_{2}=-30.8391$. For convenience, we let $\alpha_{\min }=\alpha_{\max }=0.08$ and $\beta_{\min }=$ $\beta_{\max }=0.003$; then it is easy to check that $\lambda_{1} \alpha_{\min }+\lambda_{2} \beta_{\max }=$ $0.0158>0$ which implies that condition (3) (a) of Theorem 7 is satisfied. According Theorem 7, we know that system (23) can synchronize system (45) in the sense of $\lim _{t \rightarrow+\infty} e_{1}=$ $\lim _{t \rightarrow+\infty} e_{2}=\lim _{t \rightarrow+\infty} e_{3}=0$.

The simulation results with $x_{1}(0)=6, x_{2}(0)=8, x_{3}(0)=$ $10, \hat{x}_{1}(0)=2, w(0)=6$, and $\hat{x}_{3}(0)=13$ are presented in Figure 4. From Figure 4, it is easy to see that the drive system (45) and the response system (23) are synchronized within a few seconds.
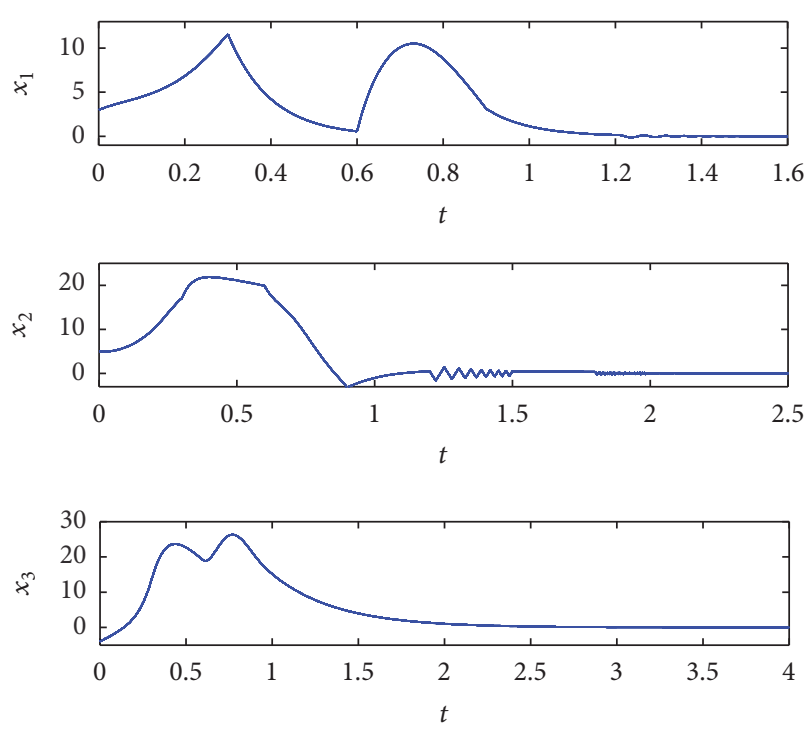

FIGURE 2: Orbits of controlled system (45) with $x_{1}(0)=3, x_{2}(0)=5$, and $x_{3}(0)=-4$.

For the sake of showing the robust of our synchronization scheme to parameter uncertainties and external disturbances, we add some parameter uncertainties and external disturbances to system (45). Thus system (45) is rewritten as

$$
\begin{aligned}
& \dot{x}_{1}=\left(10+\frac{2.5}{29}+0.1\right)\left(x_{2}-x_{1}\right)-0.2 x_{1} x_{2}, \\
& \dot{x}_{2}=\left(28-\frac{3.5}{29}-0.1\right) x_{1}-(0.9-0.1) x_{2}-x_{1} x_{3}, \\
& \dot{x}_{3}=\left(-\frac{8}{3}-\frac{0.1}{87}\right) x_{3}+x_{1} x_{2}, \\
& x_{\text {out }}=k_{1}(t) x_{1}+k_{2}(t) x_{2},
\end{aligned}
$$



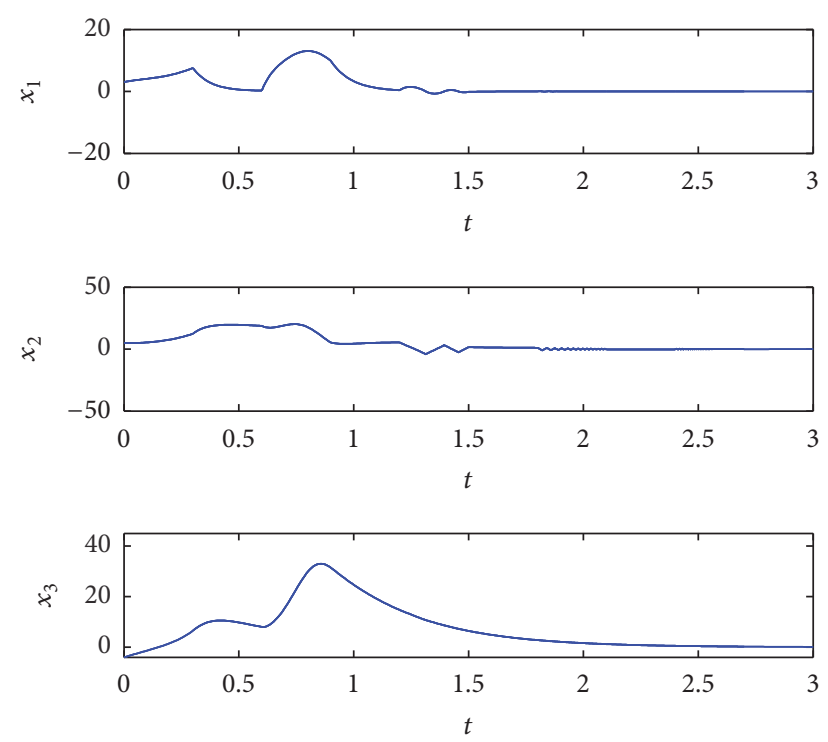

FIGURE 3: Orbits of controlled system (46) with $x_{1}(0)=3, x_{2}(0)=5$, and $x_{3}(0)=-4$.

$$
\begin{aligned}
& k_{1}(t) \\
& \quad= \begin{cases}1, & t \in\left[t_{2 m}, t_{2 m+1}\right), t_{0}=0, m=0,1,2, \ldots, \\
0, & t \in\left[t_{2 m+1}, t_{2 m+2}\right), m=0,1,2, \ldots,\end{cases} \\
& k_{2}(t) \\
& \quad= \begin{cases}0, & t \in\left[t_{2 m}, t_{2 m+1}\right), t_{0}=0, m=0,1,2, \ldots, \\
1, & t \in\left[t_{2 m+1}, t_{2 m+2}\right), m=0,1,2, \ldots,\end{cases}
\end{aligned}
$$

where $0.1\left(x_{2}-x_{1}\right)$ and $-0.1 x_{1}$ denote the parameter uncertainties, while $-0.2 x_{1} x_{2}$ represents the external disturbances. The synchronization simulation between systems (47) and (23) with the same initial conditions as in Figure 4 is presented in Figure 5. From Figure 5 it is easy to see that although in Figure 5 the synchronization between systems (47) and (23) is reached, its speed of synchronization is slower than that shown in Figure 4.

Remark 11. From Figures 3 and 5 one can conclude that the presented control and synchronization schemes are robust to some special parameter uncertainties and external disturbances. However, it should be pointed out that since in our approaches the adaptive control method is not used our schemes may not be robust to arbitrary uncertainties and external disturbances.

\section{Conclusion}

The control and synchronization of a class of chaotic systems with switched output is investigated in this paper. By using the switched output, which is assumed to be switched between the first and the second state variables, some novel criteria for
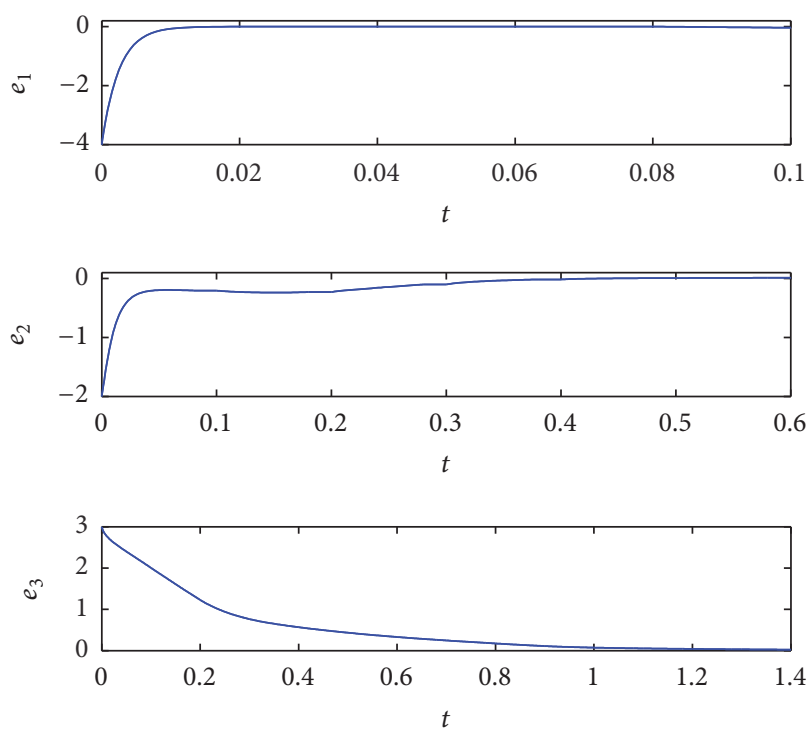

FIGURE 4: Plots of synchronization errors between systems (23) and (45).
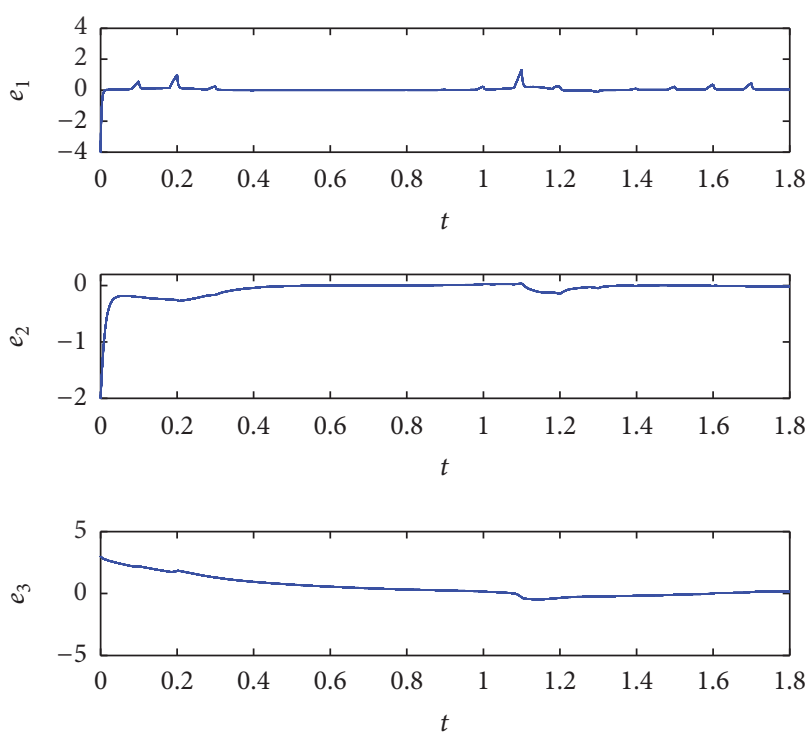

FIgURE 5: Plots of synchronization errors between systems (47) and (23).

the control and synchronization of a class of chaotic systems are proposed. The generalized Lorenz chaotic system is taken as an example to demonstrate the efficiency of the proposed approach.

It is not difficult to see that our paper has two contributions. First, we present a new model which has switched output. As it is well known that in real-life situations the transmission signals may be interrupted for various reasons which means that the output should be discontinuous variable. Since the switched output is discontinuous variable, our model is closer to the actual situation than that having continuous output variable. 
Second, the proposed model is especially suitable for secure communication. In a typical chaotic synchronization communication scheme the transmission signal is carried from the transmitter to the receiver by a chaotic signal through an analog channel. In order to mask the contents of transmission signal using chaotic signals different methods have been developed $[25,26]$. However, it has been shown that most of these approaches are not secure or have a low level of security because one can extract the encoded message signal from the transmitted chaotic signal by using different unmasking techniques [27, 28]. Using our proposed synchronization scheme to transmit signal, one can add the transmitted signal $s(t)$ to $x_{\text {out }}$ and derive

$$
\begin{aligned}
& x_{\text {out }}=k_{1}(t)\left(x_{1}+\alpha_{1} s(t)\right)+k_{2}(t)\left(x_{2}+\alpha_{2} s(t)\right), \\
& k_{1}(t) \\
& \quad= \begin{cases}1, & t \in\left[t_{2 m}, t_{2 m+1}\right), t_{0}=0, m=0,1,2, \ldots, \\
0, & t \in\left[t_{2 m+1}, t_{2 m+2}\right), m=0,1,2, \ldots,\end{cases} \\
& k_{2}(t) \\
& \quad=\left\{\begin{array}{ll}
0, & t \in\left[t_{2 m}, t_{2 m+1}\right), t_{0}=0, m=0,1,2, \ldots, \\
1, & t \in\left[t_{2 m+1}, t_{2 m+2}\right),
\end{array},\right.
\end{aligned}
$$

where $\alpha_{1} \alpha_{2} \neq 0$. From (48) it is easy to see that the transmission signal can be alternately sent to the response system which can enhance the complexity and decrease the correlation of the signals being transmitted and thereby improving the antiattack ability and antitranslated capability of the transmitted signals. Thus, our research may have significant meanings in secure communication.

Despite its advantages, our approach is not without limitations. For example, we have not taken into account the disturbances in our presented chaotic systems. It is well known that in practical applications some dynamical systems are inevitably disturbed by the noises from external circumstance. Thus, the control and synchronization of switched output chaotic systems with external disturbances is an important issue. This issue will be our research emphasis in the future.

\section{Competing Interests}

The authors declare that there is no conflict of interests regarding the publication of this paper.

\section{Acknowledgments}

This work was jointly supported by the National Natural Science Foundation of China under Grant no. 11361043 and the Natural Science Foundation of Jiangxi Province under Grant no. 20161BAB201008.

\section{References}

[1] G. Chen and X. Dong, From Chaos to Order: Methodologies, Perspectives and Applications, vol. 24 of World Scientific Series on Nonlinear Science. Series A: Monographs and Treatises, World Scientific, Singapore, 1998.

[2] E. Ott, C. Grebogi, and J. A. Yorke, "Controlling chaos," Physical Review Letters, vol. 64, no. 11, pp. 1196-1199, 1990.

[3] L. M. Pecora and T. L. Carroll, "Synchronization in chaotic systems," Physical Review Letters, vol. 64, no. 8, pp. 821-824, 1990.

[4] H. Dimassi, A. Loría, and S. Belghith, "A new secured transmission scheme based on chaotic synchronization via smooth adaptive unknown-input observers," Communications in Nonlinear Science and Numerical Simulation, vol. 17, no. 9, pp. 37273739, 2012.

[5] C. Y. Chee and D. Xu, "Chaotic encryption using discrete-time synchronous chaos," Physics Letters A, vol. 348, no. 3-6, pp. 284292, 2006.

[6] L. Kocarev and G. Jakimoski, "Logistic map as a block encryption algorithm," Physics Letters. A, vol. 289, no. 4-5, pp. 199-206, 2001.

[7] J. Zhang, X. Wang, and W. Zhang, "Chaotic keyed hash function based on feedforward-feedback nonlinear digital filter," Physics Letters A, vol. 362, no. 5-6, pp. 439-448, 2007.

[8] D. Xiao, X. Liao, and S. Deng, "Using time-stamp to improve the security of a chaotic maps-based key agreement protocol," Information Sciences, vol. 178, no. 6, pp. 1598-1602, 2008.

[9] Z. Wang, X. Huang, and H. Shen, "Control of an uncertain fractional order economic system via adaptive sliding mode," Neurocomputing, vol. 83, pp. 83-88, 2012.

[10] U. E. Vincent, "Synchronization of identical and non-identical 4-D chaotic systems using active control," Chaos, Solitons and Fractals, vol. 37, no. 4, pp. 1065-1075, 2008.

[11] D. W. Lee, W. J. Yoo, D. H. Ji, and J. H. Park, "Integral control for synchronization of complex dynamical networks with unknown non-identical nodes," Applied Mathematics and Computation, vol. 224, pp. 140-149, 2013.

[12] Z. Q. Zhu and H. P. Hu, "Robust synchronization by timevarying impulsive control," IEEE Transactions on Circuits and Systems II: Express Briefs, vol. 57, no. 9, pp. 735-739, 2010.

[13] R. Luo and Y. Zeng, "The control of chaotic systems with unknown parameters and external disturbance via backstepping-like scheme," Complexity, vol. 21, no. S1, pp. 573583, 2016.

[14] X. Xiao, L. Zhou, and Z. Zhang, "Synchronization of chaotic Lur'e systems with quantized sampled-data controller," Communications in Nonlinear Science and Numerical Simulation, vol. 19, no. 6, pp. 2039-2047, 2014.

[15] R. Luo and Y. Zeng, "The control and synchronization of a rotational relativistic chaotic system with parameter uncertainties and external disturbance," Journal of Computational and Nonlinear Dynamics, vol. 10, no. 6, Article ID 064503, 2015.

[16] H. Saberi Nik, J. Saberi-Nadjafi, S. Effati, and R. A. Van Gorder, "Hybrid projective synchronization and control of the BaierSahle hyperchaotic flow in arbitrary dimensions with unknown parameters," Applied Mathematics and Computation, vol. 248, pp. 55-69, 2014.

[17] J. M. Grzybowski, M. Rafikov, and J. M. Balthazar, "Synchronization of the unified chaotic system and application in secure communication," Communications in Nonlinear Science and Numerical Simulation, vol. 14, no. 6, pp. 2793-2806, 2009. 
[18] P. P. Singh, J. P. Singh, and B. K. Roy, "Synchronization and antisynchronization of Lu and Bhalekar-Gejji chaotic systems using nonlinear active control," Chaos, Solitons \& Fractals, vol. 69, pp. 31-39, 2014.

[19] E. E. Mahmoud, "Adaptive anti-lag synchronization of two identical or non-identical hyperchaotic complex nonlinear systems with uncertain parameters," Journal of the Franklin Institute. Engineering and Applied Mathematics, vol. 349, no. 3, pp. 1247-1266, 2012.

[20] M. Srivastava, S. K. Agrawal, and S. Das, "Adaptive projective synchronization between different chaotic systems with parametric uncertainties and external disturbances," PramanaJournal of Physics, vol. 81, no. 3, pp. 417-437, 2013.

[21] L. Runzi and W. Yinglan, "Finite-time stochastic combination synchronization of three different chaotic systems and its application in secure communication," Chaos. An Interdisciplinary Journal of Nonlinear Science, vol. 22, no. 2, 2012.

[22] E. N. Lorenz, "Deterministic nonperiodic flow," Journal of the Atmospheric Sciences, vol. 20, no. 2, pp. 130-141, 1963.

[23] S. Čelikovský and G. Chen, "On a generalized Lorenz canonical form of chaotic systems," International Journal of Bifurcation and Chaos, vol. 12, no. 8, pp. 1789-1812, 2002.

[24] J. Lü, G. Chen, D. Cheng, and S. Celikovsky, "Bridge the gap between the Lorenz system and the Chen system," International Journal of Bifurcation and Chaos in Applied Sciences and Engineering, vol. 12, no. 12, pp. 2917-2926, 2002.

[25] K. Murali, "Digital signal transmission with cascaded heterogeneous chaotic systems," Physical Review E, vol. 63, no. 1, Article ID 016217, 2001.

[26] M. Itoh, "Spread spectrum communication via chaos," International Journal of Bifurcation and Chaos in Applied Sciences and Engineering, vol. 9, no. 1, pp. 155-213, 1999.

[27] K. M. Short, "Unmasking a modulated chaotic communications scheme," International Journal of Bifurcation and Chaos in Applied Sciences and Engineering, vol. 6, no. 2, pp. 367-375, 1996.

[28] G. Pérez and H. A. Cerdeira, "Extracting messages masked by chaos," Physical Review Letters, vol. 74, no. 11, pp. 1970-1973, 1995. 


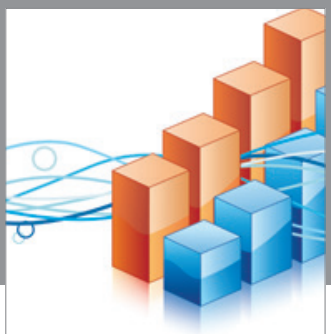

Advances in

Operations Research

vatem alat4

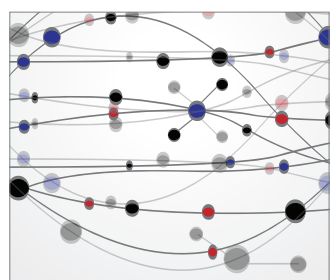

\section{The Scientific} World Journal
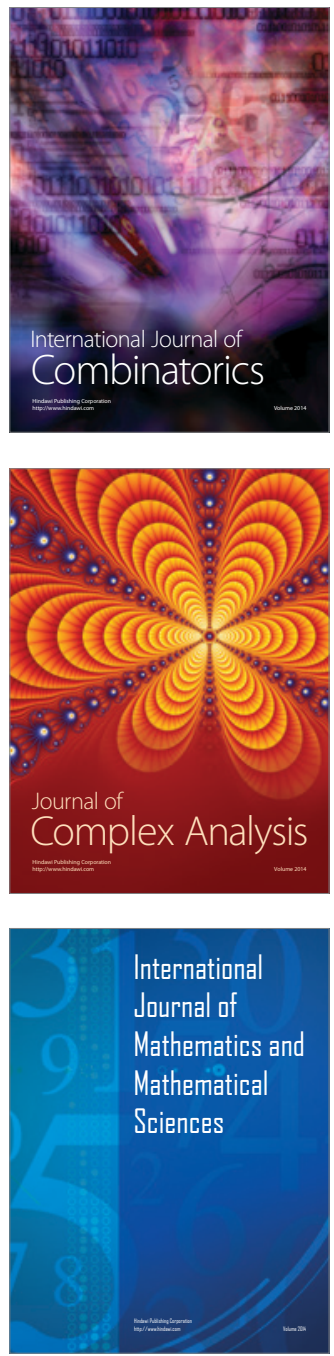
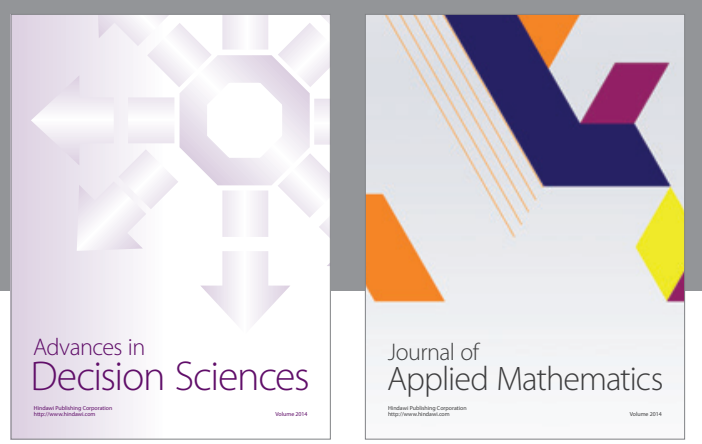

Algebra

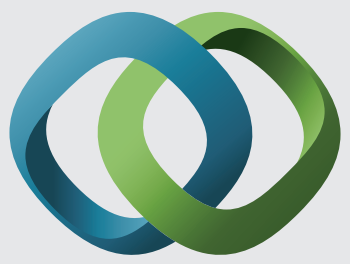

\section{Hindawi}

Submit your manuscripts at

https://www.hindawi.com
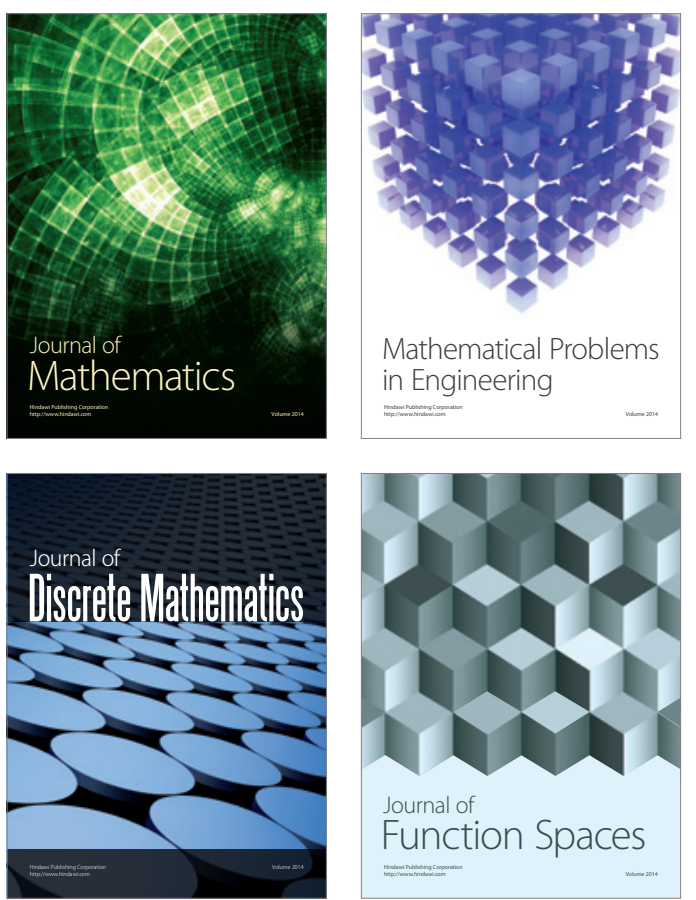

Mathematical Problems in Engineering
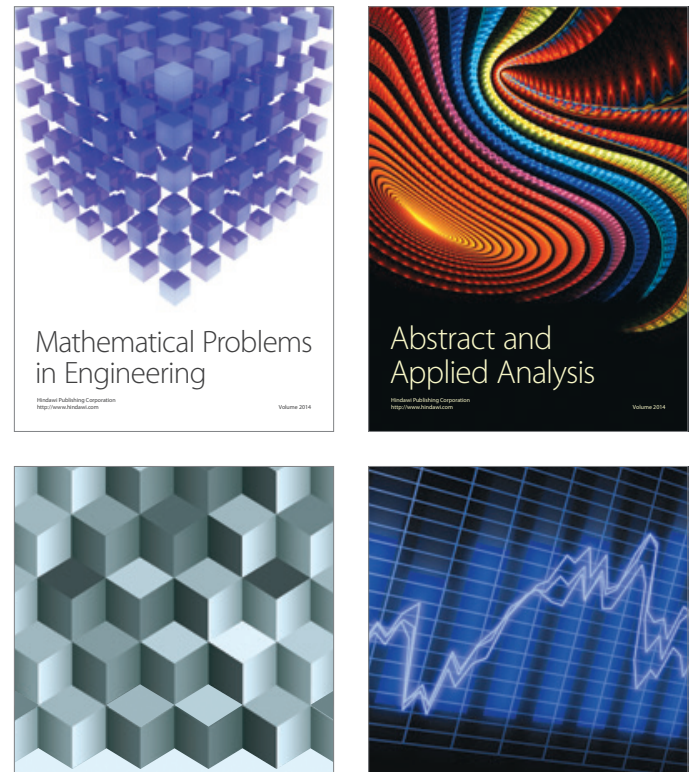

Journal of

Function Spaces

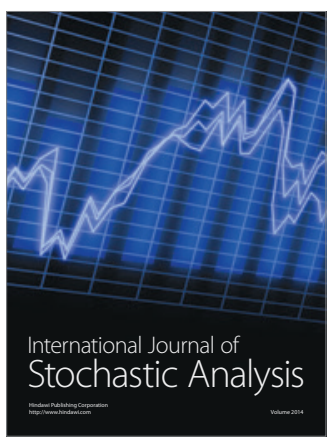

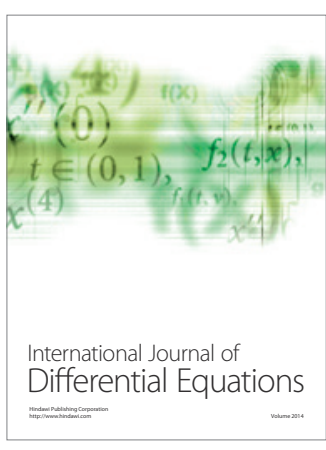
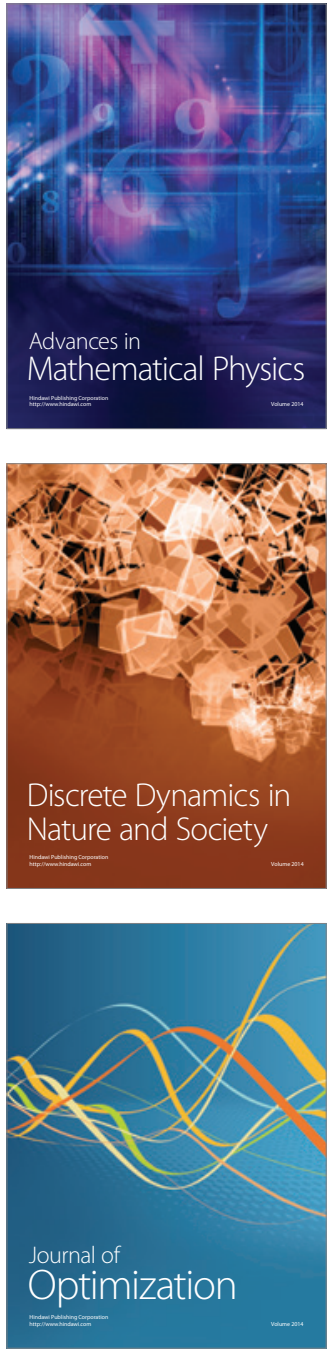